\section{Informations}

Orthop Traumatol (1991) 1:270

Juin 1992

\section{CEPUR Vittel}

Imagerie Clinique du Rachis

Paris, 10, 11 et 12 juin 1992

organisé par M.G. Dupuis et Ph. Peetrons

sous la responsabilité scientifique de J.L. Dietemann

sous la présidence de L. Jeanmart et A. Wackenheim

Informations et inscription : Dr. M.G. Dupuis, Service de Radiologie, Centre de Traumatologie et d'Orthopédie, 10, avenue Baumann, F67403 Illkirch Cedex, Tél. 886733 33, Fax 88674515

\title{
Septembre 1992
}

\section{International post-graduate course}

Bone circulation in normal and pathological conditions

Brussels, Belgium, September 25 and 26, 1992

organized by ARCO (Association Research Circulation Osseous)

Informations : $\operatorname{Pr}$. A. Schoutens, Service de Médecine Nucléaire, Hôpital Universitaire Erasme, 808 route de Lennik, B-1070 Brussels, Belgium, Tel. 3225553 300, Fax 3225554405 